\title{
REVUE NUMISMATIQUE
}

Dirigée par

C. Morrisson, M. Amandry,

M. Bompaire, 0. Picard
Secrétaires de la rédaction

Fr. Duyrat, A. Hostein,

J. Jambu

2013

(170 volume) 


\title{
COMITÉ DE PUBLICATION
}

\section{DIRECTEURS}

\author{
$\mathrm{M}^{\mathrm{me}}$ Cécile Morrisson, MM. Michel Amandry, \\ Marc Bompaire, Olivier Picard
}

SECRÉTAIRES DE LA RÉDACTION

\author{
Articles \\ $\mathrm{M}^{\mathrm{me}}$ Frédérique Duyrat \\ M. Jérôme Jambu (jeromejambu@noos.fr) \\ Comptes rendus \\ M. Antony Hostein (hosteinantony@yahoo.fr)
}

COMITÉ DE LECTURE

\begin{abstract}
Michael Alram, Jean Andreau, Philip Attwood, Gérard Aubin, François Baratte, Patrice Baubeau, Cécile Bresc, François de Callataÿ, Jean-Pierre Callu, Michel Christol, Yves Coativy, Michel Dhénin, Sylviane Estiot, Stefan Heidemann, Jérôme Jambu, Xavier Loriot, Marie-Christine Marcellesi, Jens Christian Moesgaard, Sylvia Nieto-Pelletier, Michel Pastoureau, Séléné Psoma, Andrea Saccocci, Thierry Sarmant, François Thierry, Lucia Travaini, Benedikt Zäch.
\end{abstract}

La Revue numismatique paraît annuellement. Elle est la propriété de la Société française de numismatique qui en est l'éditeur et en assure le service à tous ses membres à jour de cotisation pour l'année concernée, lors de sa parution. La cotisation a été fixée pour 2013 à $50 €$ et $55 €$ pour les membres résidant à l'étranger.

\author{
Société française de numismatique \\ 58, rue de Richelieu F-75002 Paris \\ http://www.sfnum.asso.fr \\ La Revue numismatique est également diffusée par \\ la Société d'édition «Les Belles Lettres» \\ 95 Boulevard Raspail, F-75006 Paris \\ Tél. : 01443984 20, Fax : 0145449288.
}

Les abonnements sont payables à la Société d'édition «Les Belles Lettres » Compte chèque postaux Paris $33657 \mathrm{P}$.

Le champ couvert par la Revue numismatique comprend la numismatique et l'histoire monétaire et s'étend à l'archéologie, l'histoire économique, l'histoire de l'art ainsi qu'à l'épigraphie, la sigillographie ou la glyptique dans leurs rapports avec l'étude des monnaies, médailles et documents monétiformes.

La Revue recherche des études de haut niveau et de première main, publication de documents nouveaux ou nouvelle interprétation de documents connus. Les articles sont retenus en fonction de leur qualité scientifique et de l'intérêt du document présenté. Les rubriques de la Revue sont indicatives et correspondent aux divisions historiques traditionnelles : numismatique celtique, grecque, romaine, byzantine, médiévale, moderne et contemporaine, orientale, médailles et jetons, histoire de la numismatique et des collections. Des notes synthétiques faisant le point sur une question ou un débat ont leur place dans les Miscellanea (la Société française de numismatique préférant réserver la publication des articles brefs au Bulletin de la Société française de numismatique).

Les langues admises sont, outre le français, l'allemand, l'anglais, l'espagnol et l'italien.

Les manuscrits complets et conformes aux instructions aux auteurs doivent être remis au secrétariat le $1^{\text {er }}$ juillet de l'année qui précède la parution. Après avoir été confiés à plusieurs rapporteurs et examinés par le comité de lecture, ils sont définitivement retenus lorsque le conseil de gestion de la Revue numismatique se réunit, en janvier, pour adopter le budget de la Revue qui paraît dans l'année.

La Revue ne rend compte que des ouvrages qui sont adressés au secrétariat avec la mention «Revue numismatique». Les ouvrages sont remis à des spécialistes proposés par les directeurs au Comité de lecture. La publication rapide dans le bulletin bibliographique ne doit pas nuire au caractère informatif et critique des comptes rendus et il est possible de rendre compte simultanément et synthétiquement de plusieurs ouvrages.

La Revue numismatique se réserve le droit de refuser toute publicité sans avoir à fournir de motif à sa décision. 


\title{
La circulation de monnaie carolingienne dans la péninsule ibérique. À propos d'un denier de l'atelier de Roda ${ }^{1}$
}

\begin{abstract}
Résumé - Les trouvailles de monnaies carolingiennes dans la péninsule ibérique sont peu abondantes, surtout pour les pièces frappées dans la Marche d'Espagne. Cet article présente l'état des connaissances sur cette question à propos d'un fragment de denier carolingien trouvé dans les fouilles archéologiques du site d'El Tolmo de Minateda (Hellín, Albacete, Espagne). Il s'agit d'une émission de Charlemagne de l'atelier de Roda, situé dans la Marche d'Espagne, atelier dont on ne connaissait jusqu'à maintenant qu'une seule pièce pour ce souverain franc. Le contexte stratigraphique de ce denier apporte une donnée intéressante sur le moment de son utilisation et en fait un des plus anciens exemples de la circulation de monnaies carolingiennes dans la Péninsule ibérique.
\end{abstract}

Mots clés - Numismatique, monnaies carolingiennes, circulation monétaire, péninsule ibérique, atelier de Roda, Tolmo de Minateda.

Summary - Carolingian coin finds in the Iberian peninsula are scant, especially those minted in the area known as the Marca Hispanica. This article offers a revision of this topic, starting from the find of a Carolingian denier fragment during the archaeological excavations in El Tolmo de Minateda (Hellín, Albacete, Spain). It is a coin of Charlemagne minted in the Marca mint of Roda. Until now only one coin of this king from this mint was known. The stratigraphic context of the Carolingian denier carries interesting evidence about the date of its use, and suggests that it is one of the earliest examples of Carolingian coin circulation in the Iberian Peninsula.

Keywords - Numismatic, Carolingian coins, Monetary circulation, Iberian peninsula, Roda mint, Tolmo de Minateda.

On compte très peu de découvertes de pièces carolingiennes dans la péninsule ibérique. Cette rareté se fait plus évidente encore au niveau de la zone frontalière connue sous la dénomination de Marche d'Espagne, territoire situé au sud des Pyrénées qui fut dominé par les souverains francs ${ }^{2}$. Dans cette zone, on pourrait

* Área de Arqueología, Universidad de Alicante.Courriel : carolina.domenech@ua.es.

1. Je souhaite remercier Cécile Morrisson et Patrice Cressier d'avoir eu la gentillesse de surveiller la traduction de ce texte. Je remercie également Maria Clua pour ses suggestions; et l'équipe archéologique du Tolmo de Minateda, spécialement Víctor Cañavate et Sonia Gutiérrez, sans qui ce travail n'aurait pas été possible. Le texte a été réalisé dans le cadre du projet de recherche HAR 2009-11441 du Ministerio de Ciencia e Innovación (MICINN).

2. Bien que la dénomination Marche d'Espagne ait été discutée, nous utilisons ce mot dans ce texte dans son sens géographique pour nous référer aux territoires situés au sud des Pyrénées qui ont été incorporés à l'Empire carolingien à la fin du vIII' siècle et au début du IXe siècle. 
s'attendre à davantage de découvertes de monnaies carolingiennes, mais elles y sont plus rares, et plus particulièrement en ce qui concerne les pièces frappées dans les ateliers de la Marche ${ }^{3}$.

Les premières découvertes connues de monnaies carolingiennes dans la péninsule ibérique ne datent que des années 50 du siècle dernier. C'est à cette époque qu'ont été publiés les premiers exemplaires, se résumant à quelques pièces seulement, qui se trouvaient, en petit nombre, dans deux trésors de monnaies arabes de la période émirale (de la conquête de 711 à la constitution du califat omeyade de Cordoue en 929). Le premier a été mis au jour dans le quartier Sagrada Familia de Cordoue ${ }^{4}$. L'autre a été retrouvé dans la péninsule ibérique, mais on ignore où exactement ${ }^{5}$. Comme nous le verrons plus loin, cette association de monnaies carolingiennes et de monnaies arabes est attestée dans d'autres lieux du territoire européen.

Il faut attendre les années 1980 et les travaux d'A. Balaguer et de M. Crusafont pour avoir connaissance des toutes premières découvertes dans les territoires de la Marche d'Espagne. En 1984, A. Balaguer publiait celles du territoire catalan, ainsi qu'une dizaine d'autres, faites dans la péninsule ibérique et les Îles Baléares ${ }^{6}$.

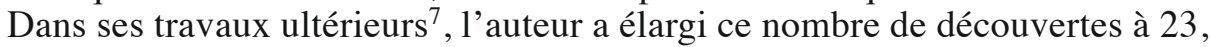
auxquelles nous pouvons ajouter aujourd'hui quelques autres, fruits du hasard pour certaines et, pour d'autres, de travaux archéologiques ${ }^{8}$.

A. Balaguer et M. Crusafont ont également montré que parmi les monnaies découvertes, très peu de pièces ont été frappées au sein de la Marche d'Espagne, et ce malgré la présence dans cette zone de plusieurs ateliers actifs. À l'époque de Charlemagne, ce territoire en comptait quatre : à Rosas, Ampurias, Gérone et Barcelone. À l'époque de Louis le Pieux, on n'en comptait plus que trois, l'atelier de frappe de Gérone ayant disparu. Cette réduction continua sous le règne de Charles le Chauve, pour lequel on ne connaît plus que des monnaies frappées à Barcelone ${ }^{9}$. Les premières monnaies connues de ces ateliers n'ont pas été retrouvées sur le territoire hispanique, mais en dehors. Les deux deniers, d'Ampurias et de Gérone, découverts à Duurstede (Pays-Bas), et les pièces de Louis le Pieux frappées à Ampurias et Barcelone, que renfermaient les trésors français de Belvezet

3. Une thèse traitant de l'étude numismatique de ce territoire et de ses ateliers est actuellement en cours de préparation ; elle pourra apporter des innovations à cet égard. Je remercie P. Sénac de cette information.

4. SANTOS 1956.

5. LAFAURIE 1952, p. 94 suggère que cette découverte a probablement eu lieu dans le comté de Barcelone ou de Gérone.

6. BALAguer 1984.

7. BALAGUER 1987-1988a-b et 1999.

8. Voir par exemple Crusafont, Joanot 2009, Marot 1999, Clua 2002, 2008, ainsi que la pièce que nous présentons ici-même.

9. Voir les ateliers et les types frappés à chacun d'eux dans BALAGUER et al. 1979, BALAGUER 1999, p. 29 et CRUSAfont 2009, p. 321-322. 
et Apremont Le Veuillin, sont les premières pièces de provenance certaine qui ont été publiées. En territoire catalan, seules 5 pièces frappées dans les ateliers de la Marche d'Espagne ont été découvertes : deux oboles de Charles le Chauve retrouvées dans les environs de Barcelone ${ }^{10}$, une autre obole de l'atelier de Roda de Louis le Pieux provenant de Rosas ${ }^{11}$, un denier attribué au même roi et de même origine $^{12}$, et un denier frappé à Roda attribué à Charlemagne apparu à Ampurias ${ }^{13}$. Dans la péninsule ibérique, jamais encore une pièce carolingienne issue d'un atelier hispanique n'avait été retrouvée en dehors du territoire sous domination franque, jusqu'à la mise au jour de la monnaie que nous étudions ici sur l'établissement du haut Moyen Âge d'El Tolmo de Minateda.

Une autre question intéressante sur laquelle il convient de s'arrêter est celle de la chronologie de ces monnaies carolingiennes découvertes dans la péninsule ibérique. Il s'agit essentiellement d'émissions réalisées à partir du règne de Louis le Pieux (814-840). On ne connaissait encore aucune monnaie frappée à l'époque de Charlemagne jusqu'à ce que M. Crusafont publie en 1983 une monnaie de l'atelier de Roda attribuée à celui-ci. Ce même auteur révélait l'existence d'une seconde pièce de ce souverain de l'atelier de Toulouse, retrouvée fortuitement à Anserall, tout près du monastère de Sant Serni de Tavèrnoles dans l'Alto Urgell ${ }^{14}$. Dans les deux cas, leur attribution à Charlemagne a été fondée sur des critères stylistiques et historiques. À ces critères s'ajoute, pour la monnaie que nous présentons, l'argument stratigraphique. Les fouilles archéologiques réalisées de façon systématique sur le site d'El Tolmo de Minateda (Hellín, Albacete) ont donné lieu à la mise au jour d'un fragment de monnaie de Charlemagne de l'atelier de Roda, attestant ainsi pour la première fois de la circulation sur le territoire d'al-Andalus de monnaies de Charlemagne.

\section{El Tolmo de Minateda : une cité du haut Moyen Âge}

El Tolmo de Minateda (Hellín, Albacete) est un site archéologique du sud-est de la péninsule ibérique ${ }^{15}$ (figure 1). Il est établi sur un emplacement stratégique privilégié qui contrôle la voie naturelle de communication vers l'intérieur de

10. CRusafont 1980.

11. BÉNEZET et al. 2011.

12. Cette découverte publiée par F. Mateu en 1946 avait suscité le doute chez certains chercheurs qui la considéraient comme peu fiable, mais elle figure finalement dans la bibliographie la plus récente. Voir p. 397.

13. CRUSAFont 1983.

14. Crusafont, Joanot 2009, p. 237. Type Prou 801 variant.

15. Le Tolmo de Minateda a commencé à être fouillé en 1988 dans le cadre d'un projet de recherche financé par la Dirección General de Educación, Ciencia y Cultura, de la Junta de Comunidades de Castilla-La Mancha. À ce projet participent l'Université d'Alicante et le Musée d'Albacete. Il a été déclaré Parc Archéologique de la Province d'Albacete en 2012 (ABAD et al. 2012) par le décret 81/2012 du 26/04/2012 [2012/6577]. 
la Péninsule et la zone côtière du Sud-Est, au croisement d'anciennes routes de communication dûment documentées à partir de milliaires romains ${ }^{16}$.

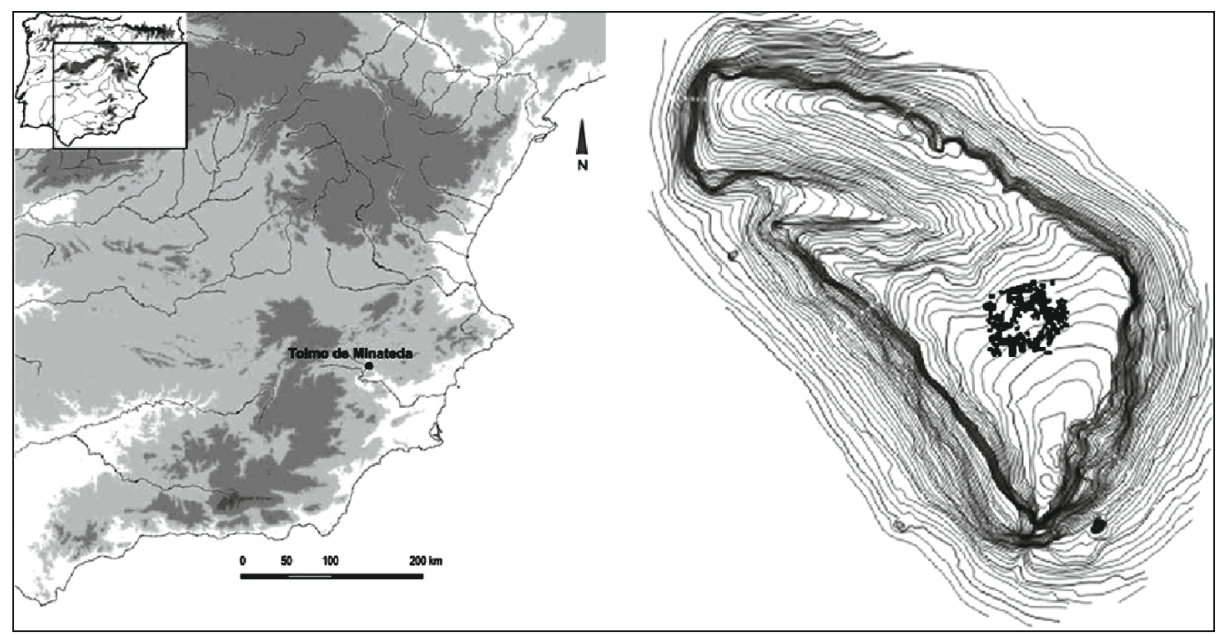

Figure 1 - Carte de situation d'El Tolmo de Minateda et emplacement du quartier emiral.

La localisation de cette cité a permis des établissements humains au cours du temps. Nous constatons d'importants vestiges qui nous signalent une longue occupation. La séquence d'occupation d'El Tolmo de Minateda a son origine au moins à la période néolithique, si nous tenons compte de la chronologie du sanctuaire rupestre de l'abri de Minateda, dans lequel nous pouvons trouver des peintures de style levantin. Ces établissements ne sont pas continus dans le temps, puisque nous avons documenté des ruptures dans l'habitat. Nous pouvons constater un établissement de l'âge du bronze, datant du IIIe millénaire av. J.-C. et une phase préromaine qui correspond à un oppidum ibérique transformé en municipium romain de la période d'Auguste ${ }^{17}$. Cette ville a été abandonnée autour du IIe siècle av. J.-C., et n'a pas été réoccupée jusqu'à la période wisigothique quand, à la fin du vie siècle ou au début du vIIe siècle, dans le cadre du conflit qui a opposé les Wisigoths et les Byzantins, une importante activité urbaine de construction s'y est manifestée. Les données archéologiques montrent l'ampleur de l'intervention urbaine wisigothe qui peut être considérée comme un projet ex novo. À ce moment là, toute la surface de la colline s'urbanise et on observe la création d'un grand complexe

16. Voir SiLlières 1982 et 1990. Il s'agit de la chaussée romaine qui reliait Complutum (Alcalá de Henares, Madrid) à Carthago Nova (Carthagène) qui croise en ce point la voie transversale qui, depuis Valence, mène à la haute Andalousie (ABAD et al. 1998).

17. АвAD 1996. 
monumental religieux et administratif constitué par une basilique et un palatium dans la partie haute de la ville, autour de laquelle se développe un cimetière $a d$ sanctos, tandis qu' une nouvelle muraille est bâtie dans l'accès principal, englobant les ruines de fortifications ibériques et romaines ${ }^{18}$. Son emplacement stratégique dans le principal axe de communication entre Toletum (Toledo), siège royal wisigothique et Carthago Spartaria (Carthagène), capitale byzantine, a déterminé son érection comme centre épiscopal. Actuellement est en effet communément acceptée l'identification d'El Tolmo de Minateda au siège épiscopal d'Eio/Elo, créé par la monarchie wisigothique au début du VIIe siècle, conjointement avec celui de Begastri, pour intégrer les territoires dépendant des sièges d'Ilici et de Carthago Spartaria qui restaient encore sous domination byzantine ${ }^{19}$.

La ville wisigothique était encore habitée au moment de la conquête araboberbère de 711, et a subsisté durant la première période islamique. Jusqu'à la fin du viIIe siècle ou au début du siècle suivant, la partie monumentale où se trouvaient la basilique et le palatium wisigothiques a connu une importante évolution urbaine planifiée : il a été construit un quartier musulman sur le groupe épiscopal. Sous son nom arabisé, Madīnat Iyyuh qui est resté dans le toponyme Minateda, la ville est mentionnée dans le célèbre Pacte de Teodomiro, pacte de soumission signé l'an 713 par 'Abd al-'Azīz Ibn Mūsà et le dux Teodomiro (Theudimer). Ce pacte, le seul connu en détail pour tout al-Andalus, signifiait la soumission d'une vaste région du sud-est de l'Hispania, qui a été désignée à partir de ce moment-là dans les sources arabes comme Kūra de Tudmīr (la province de Teodomiro). L'établissement fut habité sans interruption jusqu'au IXe siècle ${ }^{20}$. Lors de son utilisation, ce quartier a subi quelques rénovations et réformes traduisant d'un côté des changements fonctionnels, et d'un autre côté un processus de concentration des espaces domestiques qui tendent à devenir de plus en plus complexes ${ }^{21}$. La cité est complètement abandonnée à une date non précisée du IXe siècle, avant l'instauration du Califat du Cordoue en 929 apr. J.-C. ${ }^{22}$.

La plupart des monnaies collectées sur le site d'El Tolmo de Minateda proviennent des couches du haut Moyen Âge. Elles ont été exhumées principalement dans des contextes d'occupation, de transformation, d'abandon et de destruction ${ }^{23}$. Les plus nombreuses sont les monnaies romaines, les bronzes du Bas-Empire, du III et surtout du Ive siècle apr. J.-C., qui ont circulé, déjà très usés, dans la cité du haut Moyen Âge.

18. GutiérRez, CÁnovas 2009.

19. GuTIÉRREZ 2000 ; GuTIÉRREZ et al. 2005.

20. GuTIÉRREZ 2011.

21. Gutiérrez 2008 ; Gutiérrez, Cañavate 2010.

22. Ces niveaux du haut Moyen Âge du site archéologique ont donné lieu à une vaste bibliographie, détaillée dans les dernières publications, particulièrement GuTIÉRREZ 2011 et ABAD et al. 2012.

23. Sur les monnaies d'El Tolmo de Minateda et leur contexte voir DomÉNECH, GutiérRez 2005, 2006 ; DomÉNECH 2009, 2010 ; GutIÉRREZ, DoMÉNECH sous presse. Ces monnaies sont conservées au Musée d'Albacete. 
Parmi les monnaies du haut Moyen Âge, figure un bronze byzantin de la série croix/delta avec une valeur de 4 nummi (planche I, 7). Il a été identifié par M. Lechuga comme une émission locale de Carthago Spartaria, la capitale byzantine, d'où proviennent la plupart des pièces connues de cette série. Ces petits bronzes byzantins ont été datés par les contextes archéologiques entre la seconde moitié du VIe siècle et début du vII ${ }^{e}$ siècle ${ }^{24}$. Ont été exhumés également six tremisses wisigoths d'Ervigio, d'Égica, de Witiza et du règne commun d'Égica et Witiza, tous datés d'entre les années 680 et 710 apr. J.-C. (planche I, 1-6) ${ }^{25}$.

Les monnaies islamiques récupérées jusqu'à présent sont au nombre de vingtquatre, dont dix sont des dirhams d'argent et le reste sont des fulus de cuivre ${ }^{26}$. La plupart des dirhams appartiennent aux émissions d'al-Andalus, principalement de la première moitié du IX ${ }^{\mathrm{e}}$ siècle ${ }^{27}$. Nombre d'entre eux sont apparus coupés en fragments d'un quart de pièce. En plus ont aussi été trouvés un dirham abbasside au nom du calife Hārūn al-Rashīd, daté d'entre les années 795 et 802 apr. J.-C., et le fragment d'une monnaie carolingienne que nous décrivons ci-dessous.

\section{La monnaie carolingienne et son contexte}

Pendant la fouille des niveaux islamiques d'El Tolmo de Minateda a été récupéré un fragment d'environ la moitié d'une monnaie d'argent qui s'est avéré être un denier carolingien (figures 2 et 3). Il s'agit d'une émission de Roda, atelier de frappe situé dans la Marche d'Espagne, dont on ne connaissait jusqu'alors qu'une seule pièce, trouvée près d'Ampurias et publiée par M. Crusafont ${ }^{28}$ (figure 4). Cet auteur a démontré qu'il s'agissait d'une monnaie de Charlemagne.

Le droit de la monnaie qui fait l'objet de notre étude se présente dans un très mauvais état de conservation qui ne permet de lire qu'une partie de la légende : $+\mathrm{CA}[. ..] \mathrm{FR}$; tandis qu'au revers on trouve une partie du monogramme KAROLVS entouré par le nom de l'atelier de frappe: [+] RO • D + [DA].

Le revers de la monnaie présente les lettres très espacées: dans la moitié conservée de la pièce n'apparaissent que trois des lettres correspondant à l'atelier de frappe. Il est donc tout à fait possible que dans l'autre moitié figurent les deux manquantes et la croisette initiale, auxquelles peut-être, pourrait être ajoutée quelque ponctuation entre les lettres. Cette caractéristique du large espacement se produit également dans l'autre exemplaire connu de cet atelier attribué à Charlemagne. Tous les deux ont été frappés avec des coins différents, comme on peut le voir par la dissemblance de graphie, et par la position distincte du point qui dans l'exem

24. Lechuga 2000 ; Lechuga, MÉndez 1991.

25. DomÉnech 2002 et DomÉNeCH, GuTIÉRREZ 2005.

26. Quelques fulus, planche I, nos 13-18.

27. Sur leur contexte et leur fragmentation voir DomÉNECH, GutiÉrREz 2006.

28. CRusafont 1983, p. 125-135. 

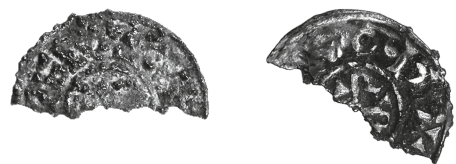

Figure 2 - Denier de Charlemagne de l'atelier de Roda trouvé dans les fouilles d'El Tolmo de Minateda.
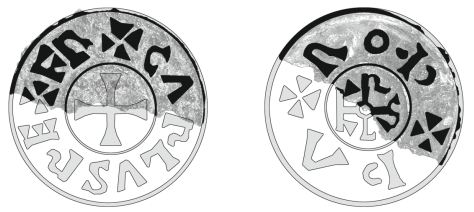

Figure 3 - Proposition de restitution de la monnaie d'El Tolmo de Minateda.
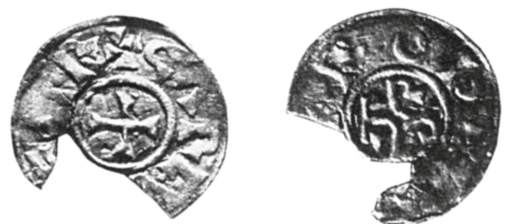

Figure 4 - Monnaie attribuée à Charlemagne et frappée à l'atelier de Roda publiée par M. Crusafont (2009, p. 321, n 1803).

plaire publié par M. Crusafont sépare le $\mathrm{R}$ du $\mathrm{O}$, tandis que, dans notre monnaie, il se situe entre le $\mathrm{O}$ et le $\mathrm{D}^{29}$. Néanmoins les deux pièces présentent quelques similitudes épigraphiques comme une croix après le premier $\mathrm{D}$.

La pièce présente un module de $20 \mathrm{~mm}$ et un poids de $0,5 \mathrm{~g}$. Étant donné qu'il s'agit d'un fragment d'environ une demi-monnaie, le poids de la monnaie complète devrait être de l'ordre de $1 \mathrm{~g}$. Ce poids, un peu faible pour les deniers carolingiens, s'adapte parfaitement à la métrologie des deniers frappés sur le territoire de la Marche d'Espagne. Selon M. Crusafont, ceux-ci échappent à l'étalon métrologique des deniers reformés carolingiens : les monnaies de Charlemagne étudiées par lui ont des poids de 1,38 $\mathrm{g}$ pour l'atelier de Gérone, $1,01 \mathrm{~g}$ pour une pièce

29. Sur l'utilisation de points et autres marques sur les monnaies de Charlemagne voir Coupland 2005, p. 218. 
d'Ampurias et 1,21 g pour celles frappées à Barcelone ${ }^{30}$. Le poids moyen pour ces trois exemplaires complets est de 1,2 g, ce que M. Crusafont a donné comme l'estimation la plus probable de leur masse bien que l'échantillon soit nettement insuffisant. Selon cet auteur, ce poids, clairement inférieur à celui des monnaies d'autres ateliers de frappe de l'empire, semble maintenir pour les émissions de la Marche d'Espagne l'étalon métrologique carolingien antérieur à la réforme de Charlemagne ${ }^{31}$. Pour l'atelier de Roda, on ne connaît pas de pièces entières : le poids de l'exemplaire incomplet trouvé à Ampurias est de $0,94 \mathrm{~g}$ ou de $0,80 \mathrm{~g}^{32}$, et la demi-monnaie d'El Tolmo de Minateda pèse $0,5 \mathrm{~g}$, soit environ $1 \mathrm{~g}$ si elle était complète.

La fragmentation de la monnaie que nous présentons a été effectuée de sorte que la coupure n'a pas un bord droit mais irrégulier, avec plusieurs saillants et entrants. Ce mode de partition est similaire à celui qui a été pratiqué sur d'autres pièces d'argent islamiques trouvées à El Tolmo de Minateda (planche I, 9-12). En effet, la plupart de ces monnaies d'argent sont des fragments, à l'exception de deux exemplaires, dont un dirham abbasside du calife Hārūn al-Rashīd (planche I, 8). La pratique de fragmentation est fréquente dans al-Andalus omeyyade, non seulement à la période califale, quand l'absence d'émissions de cuivre pourrait expliquer ce phénomène pour obtenir de la monnaie fractionnaire, mais aussi pendant l'émirat lorsqu'il y a en circulation de la monnaie de cuivre ${ }^{33}$. Cette fragmentation a porté non seulement sur les pièces anciennes, mais aussi sur des exemplaires qui avaient quitté l'atelier de frappe peu de temps auparavant, comme cela a été documenté à El Tolmo de Minateda ${ }^{34}$. Le denier carolingien étudié ici, a dû circuler conjointement avec les dirhams et s'intégrer parfaitement dans la masse monétaire en circulation de la cité. Il a été coupé sous la forme d'une fraction régulière et reconnaissable d'une demi-pièce, subissant le même processus de fragmentation que les dirhams de l'émirat omeyyade.

Cette monnaie a été trouvée dans un axe de circulation que nous avons nommé «rue du Nord», dans une couche de la période islamique datée du IXe siècle ${ }^{35}$ (figure 5). Bien que ces contextes de circulation puissent sembler peu fiables, la position de cette couche dans la séquence stratigraphique générale du site suggère qu'il s'agit de l'une des strates les plus anciennes parmi celles qui sont

30. CRUSAFont 1982, p. 26-27.

31. CRUSAFOnt 1983, p. 128.

32. Le premier chiffre est celui de la publication de 1983 où M. Crusafont donne un poids de $0,94 \mathrm{~g}$ et un module de $18,5 \mathrm{~mm}$. Dans le catalogue de 2009, où la pièce figure comme unique, le poids est $0,80 \mathrm{~g}$ et le module $22 \mathrm{~mm}$.

33. En l'absence d'émissions en or, le dirham était la plus forte valeur dans le système monétaire de l'émirat d'al-Andalus. C'était donc, la monnaie fiscale avec laquelle l'État recueillait les impôts. Sa haute valeur détermine sa dynamique circulatoire et explique la pratique de couper les pièces pour obtenir de la monnaie fractionnaire ou divisionnaire.

34. DomÉneCh, GutiÉRrez 2006, p. 360-361.

35. UE : 63277. 


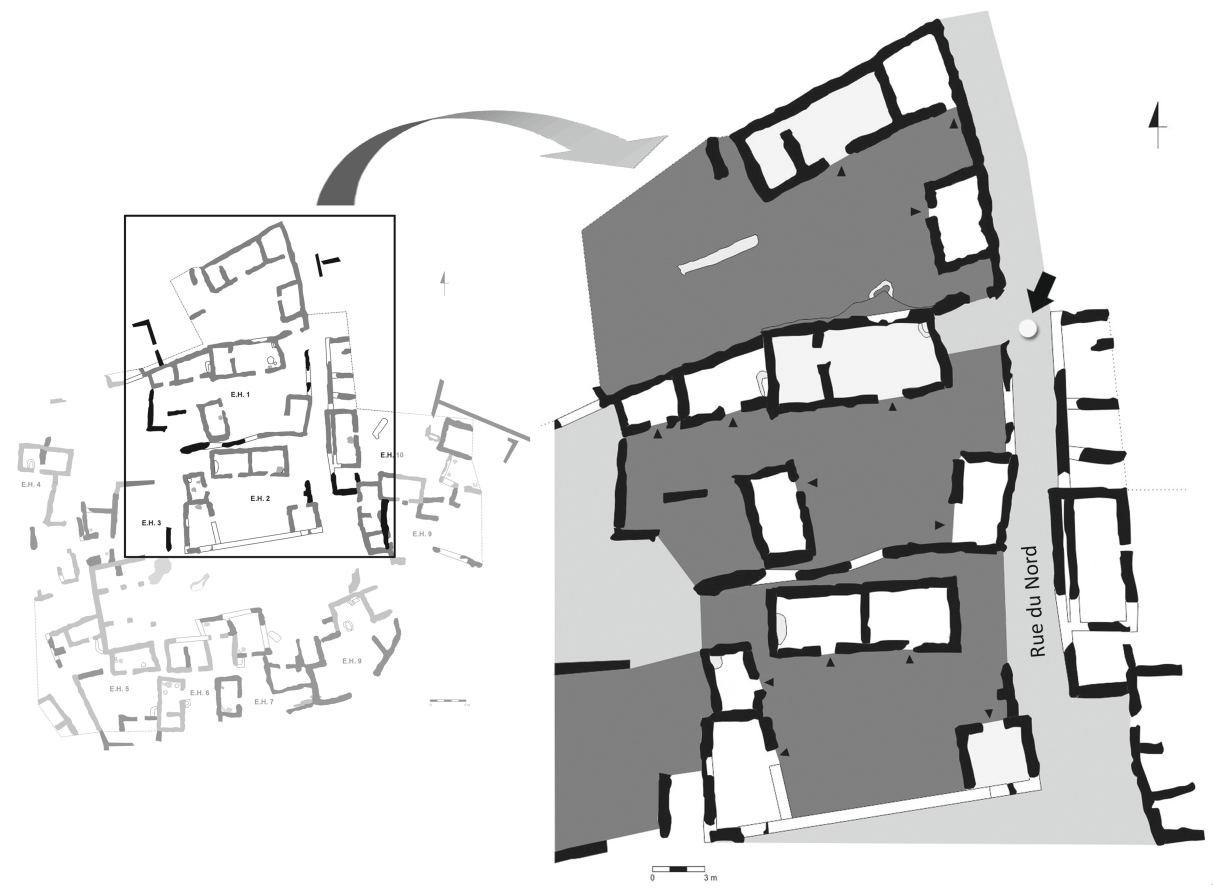

Figure 5 - Localisation de la monnaie carolingienne d'El Tolmo de Minateda.

datées du IXe siècle, proche des premières constructions du quartier islamique ${ }^{36}$. $\mathrm{Au}$-dessus de celle-ci, nous en retrouvons d'autres correspondants aux niveaux d'effondrement et d'abandon de la cité émirale de la deuxième moitié du Ixe siècle (figure 6). Si nous tenons compte du fait que les premières constructions islamiques d'El Tolmo de Minateda sont datées au plus tôt de la fin du viIIe siècle, ou plus probablement au début du Ixe siècle, on peut alors voir que la monnaie carolingienne aurait dû être perdue à un certain moment de la première moitié du Ixe siècle. Cette donnée permet de proposer son attribution à Charlemagne. La possibilité qu'il s'agisse d'une monnaie émise sous le gouvernement de Charles le Chauve est moins probable à partir du contexte, car ce monarque émit les séries au monogramme après l'édit de Pîtres de 864 et avant cette date dans les ateliers de Bourges, Melle,

36. Le contexte du matériel céramique de cette couche illustre le passage de la phase II (abandon et spoliation du complexe épiscopal, et nivellement et construction du quartier islamique) à la phase III, (occupation et abandon du quartier islamique). Voir figure 6. Sur ces matériaux céramiques voir GuTIÉRREZ et al. 2003 et Amorós et al. 2012. 


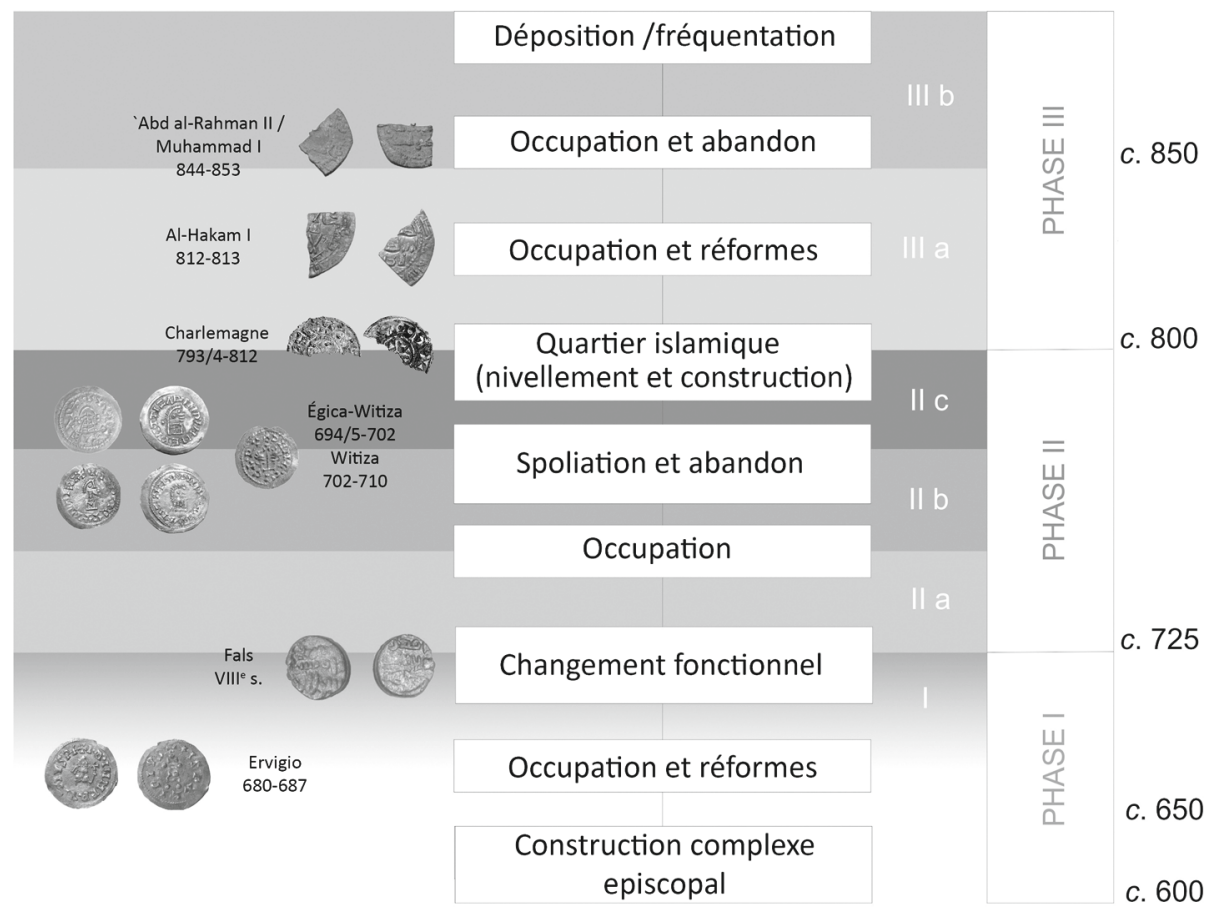

Figure 6 - Séquence stratigraphique générale d'El Tolmo de Minateda et position de la monnaie carolingienne.

Sens et Toulouse ${ }^{37}$. Avec une telle date d'émission, le fragment de monnaie n'aurait pas pu circuler dans l'établissement avant le dernier tiers du Ixe siècle (en admettant la date de 864) ou, en tout cas, la seconde moitié du Ixe siècle. Ajoutons encore que, jusqu'à présent, aucune monnaie frappée dans la seconde moitié du IXe siècle n'a été trouvée sur le site, la pièce la plus tardive étant un fragment de dirham d'al-Andalus frappé entre les années 844 et 853 apr. J.-C. et trouvé dans une couche d'utilisation de la dernière phase ${ }^{38}$. L'atelier de frappe fournit une autre donnée importante, dont il faut tenir compte : en effet, pour le règne de Charles le Chauve ne sont connues, pour le moment, que les monnaies frappées dans l'atelier de Barcelone, d'un type différent de celui au monogramme qui sont facilement différentiables de celles de Charlemagne.

37. G. Sarah (2010) a démontré que, dans ces ateliers, Charles le Chauve émit les séries au monogramme pendant la première période (840-864).

38. Ce dirham a été trouvé à l'intérieur d'une maison du quartier émiral près d'un ensemble céramique datant de la seconde moitié du IXe siècle. Voir DomÉnech, Gutiérrez 2006, p. 360-362. 
Ce contexte stratigraphique apporte encore une donnée intéressante sur le moment d'usage de ce denier carolingien. A. Canto a récemment suggéré que la chronologie d'arrivée du numéraire carolingien dans la péninsule ibérique pourrait se concentrer sur la deuxième moitié du IXe siècle apr. J.-C. . $^{39}$. Or, le contexte stratigraphique du denier carolingien trouvé à El Tolmo de Minateda laisse supposer une chronologie légèrement antérieure : la première moitié du IXe siècle. Cela nous indique qu'il s'agit d'un des exemples plus anciens de la circulation monétaire carolingienne dans la péninsule ibérique. Les données fournies par la contextualisation stratigraphique de cette pièce permettent aussi de faire quelques considérations sur le vieux problème d'attribution des émissions avec monogramme à Charlemagne ou à Charles le Chauve.

\section{Charlemagne versus Charles le Chauve}

Les pièces qui portent une croix au droit avec la légende CARLVS REX FR, et au revers le monogramme KAROLVS entouré d'une légende avec l'atelier de frappe, correspondent au type établi par Charlemagne vers 793-794. Maintenu jusqu'en 812, celui-ci fut ensuite remplacé par d'autres symbolisant l'autorité impériale ${ }^{40}$. Ce type a été repris par la suite par Charles le Chauve après l'édit de Pîtres de 864 et avant cette date dans les ateliers de Bourges, Melle, Sens et Toulouse $^{41}$. L'utilisation d'un même type par les deux monarques a soulevé de nombreux débats d'attribution à l'un ou à l'autre, et même à d'autres rois Charles leur ayant succédé.

Cette attribution s'est d'abord appuyée sur des critères stylistiques pas toujours admis $^{42}$. Mais en 1852, L. de Coster résumait les différentes positions des chercheurs à ce sujet et apportait de nouvelles informations avec la publication des monnaies retrouvées dans un lieu situé à proximité de Duurstede, aux Pays-Bas. La découverte de monnaies au monogramme dans cette ville, détruite par les vikings à l'époque de Louis le Pieux, ne laissait plus place au doute quant à l'attribution de ces émissions à Charlemagne. Parmi les pièces retrouvées près de Duurstede, il y avait deux deniers, en bon état de conservation, frappés à Gérone et à Ampurias, au sein de la Marche d'Espagne. Bien que certains chercheurs, comme M. Prou ${ }^{43}$, n'aient pas considéré cet argument comme complètement probant, avançant que

39. CANTO 2012, p. 72.

40. Selon J. Lafaurie (1976, p. 67), l'émission des monnaies à la titulature impériale commence au moment où l'empereur byzantin Michel I ${ }^{\mathrm{er}}$ reconnait le titre d'empereur à Charlemagne en 812, alors que S. Coupland (2010-1) tend à situer un peu plus tard l'émission impériale, vers 813. À l'heure actuelle, il semble peu probable que l'émission des monnaies à la titulature impériale commence dans quelques ateliers à partir du Capitulaire de Thionville de Noël 805, comme le pense Ph. Grierson (1965).

41. Voir n. 37.

42. Grierson 1965, p. 524-527, Grierson, Blackburn 1986. Plus récemment Sarah 2010.

43. Prou 1892, p. V et suiv. 
la cité n'avait pas totalement disparu,il est aujourd'hui admis que les nombreuses pièces au monogramme mises au jour à Duurstede, plusieurs découvertes récemment, sont bien des émissions de Charlemagne. Les travaux de S. Coupland ont montré que ce territoire a connu son plein essor sous les règnes de Charlemagne et de Louis le Pieux ${ }^{44}$, et a commencé à décliner considérablement à partir de $840^{45}$. Il y a quelques monnaies de Lothaire (840-855) et des imitations locales déjà présentes dès le règne de Louis le Pieux, mais à l'époque de Charles le Chauve, Duurstede ne semble pas être déjà sous domination carolingienne.

On admet actuellement une frappe sous les deux règnes et, hors contexte, il est parfois difficile de préciser l'attribution à Charlemagne ou à Charles le Chauve ${ }^{46}$. Si cette attribution a été fondée de prime abord sur des critères stylistiques pas toujours admis, dans le cas précis des deniers frappés au sein de la Marche d'Espagne, M. Crusafont s'appuyait sur des critères historiques et suivait les arguments de J. Botet pour les attribuer à Charles le Chauve ${ }^{47}$, contrairement à la thèse défendue par la majeure partie de l'historiographie française qui les attribuaient à Charlemagne. Toutefois, peu de temps après, il démontrait lui-même que ces deniers au monogramme appartenaient bien au règne de Charlemagne, fondant cette attribution sur la découverte à Duurstede de deniers au monogramme frappés à Gérone et à Ampurias, ainsi que sur la mise au jour à Barcelone d'oboles de Charles le Chauve frappées dans cette même ville, d'un type différent de celui au monogramme. Cette dernière découverte ouvrait donc de nouveau la voie aux hypothèses d'attribution des deniers au monogramme à Charlemagne ${ }^{48}$.

À ces arguments s'ajoute à présent, pour la monnaie d'El Tolmo de Minateda, l'argument stratigraphique, qui nous permet d'attribuer cette pièce à Charlemagne. Si le contexte général du site, où aucune monnaie frappée dans la seconde moitié du IXe siècle n'a été trouvée, suggère qu'il ne s'agit pas d'une émission de Charles le Chauve, le contexte particulier de la pièce, à savoir un niveau de circulation d'une rue, au-dessus de laquelle ont été documentées d'autres couches antérieures à l'abandon de la cité au milieu du IXe siècle, semble confirmer également cette théorie. Étant donnée la position stratigraphique de la monnaie dans une couche datant de la première moitié du IXe siècle, il semble peu probable qu'elle ait été frappée par Charles le Chauve, étant donné que ce monarque cède à l'évêque Frodol le tiers du bénéfice de la monnaie du comté de Barcelone en $862^{49}$, à savoir

44. Voir Coupland 1988, 2002, 2005, 2007 et 2010-2.

45. Les causes de ce déclin sont décrites dans l'ouvrage de Coupland 2010-2, p. 101-103.

46. Sur cette question voir Coupland 2005, p. 219 et Depeyrot 2008, p. 37. Récemment G. Sarah (2010) a réussi à établir de nouveaux critères pour distinguer les émissions en combinant l'étude des ateliers avec d'autres données numismatiques comme la composition des trésors et la loi des monnaies.

47. Crusafont 1980, p. 49.

48. CRUSAFont 1982, p. 27 et 1983.

49. CRUSAFont 1996, p. 57. 
deux ans avant l'édit de Pîtres que reprend les émissions du monogramme ${ }^{50}$. En fait, c'est à ce moment que commencent à être frappées au sein de la Marche d'Espagne les pièces dites de transition avec un monogramme non déchiffré et dont l'autorité émettrice ne semble pas être celle des rois francs ${ }^{51}$.

L'argument stratigraphique visant à déterminer l'attribution des pièces au monogramme à Charlemagne ou à Charles le Chauve suscité par les découvertes de Duurstede et utilisé récemment sur les trouvailles de Paule (Finistère) ${ }^{52}$, s'avère significatif dans le cas d'El Tolmo de Minateda. En ce qui concerne les autres découvertes, dont on ignore le contexte archéologique, il semble difficile de les attribuer à l'un ou à l'autre. C'est le cas pour plusieurs découvertes pyrénéennes, notamment les deniers émis par l'atelier Metvllo trouvés à Llívia (Cerdagne), Vinçà (Conflent) ou dans la zone de Gérone ${ }^{53}$. Ils ont tous été attribués à Charles le Chauve, sur des critères de style. Toutefois, comme A. Balaguer le précise à juste titre, il ne faut pas écarter l'hypothèse de leur appartenance au règne de Charlemagne, notamment dans le cas des deux premiers ${ }^{54}$. Il en va de même pour les deniers retrouvés dans les trésors de Castell Roselló à Perpignan ou de Sagrada Familia à Cordoue. Le fait que l'atelier de Roda ne semble pas avoir émis monnaie sous le règne de Charles le Chauve est une autre question qu'il convient de considérer.

\section{L'atelier de Roda}

Il est aujourd'hui admis que cet atelier, figurant sur les monnaies carolingiennes comme RODDA, se trouvait à l'emplacement de l'ancienne Rosas (figure 7). Toutefois, sa localisation a longtemps fait l'objet de nombreuses hypothèses, l'une d'elles le situant même en territoire franc au nord des Pyrénées ${ }^{55}$.

L'existence d'un atelier portant le nom de Rodas en territoire catalan à l'époque wisigothe ${ }^{56}$ et le maintien des ateliers wisigoths à l'époque carolingienne ${ }^{57}$ ont permis de faire le rapprochement entre la Roda wisigothe et la carolingienne.

50. Bien qu'à certains ateliers comme Sens, Melle, Bourgues et Toulouse, ce type commence à être émis a partir de 840. Voir SARAH 2010.

51. Clua 2008, p. 79. Selon M. Crusafont (2002, p. 275) ces nouveaux types ont été introduits par l'évêque Frodol. Voir aussi Sanahuja 2006, Crusafont 2007.

52. Voir SARAH et al. 2011.

53. BaLAGUER 1987-1988b, nos 20-22, p. 317-320.

54. BALAGUER 1987-1988b, p. 317.

55. A. Engel et R. Serrure (1890, p. 127) le situaient à La Rodde française, tandis que J. Botet (1908-1911, p. 12) évoquait sa possible localisation dans le comté de Rodez.

56. À l'époque wisigothe, deux ateliers aux noms très similaires ont frappé de la monnaie : Roda en Bétique et Rodas en Tarraconaise. Pour les ateliers portant le nom de Roda à l'époque wisigothe, voir BALAGUER 1981-1983 et 1983, et VICO et al. 2006, p. 178 et 212.

57. Le seul atelier de la Marche d'Espagne qui ne compte pas un passé wisigoth est celui d'Ampurias. Pour en savoir plus à ce sujet, voir BALAGUER 1981-1983 et 1983. 


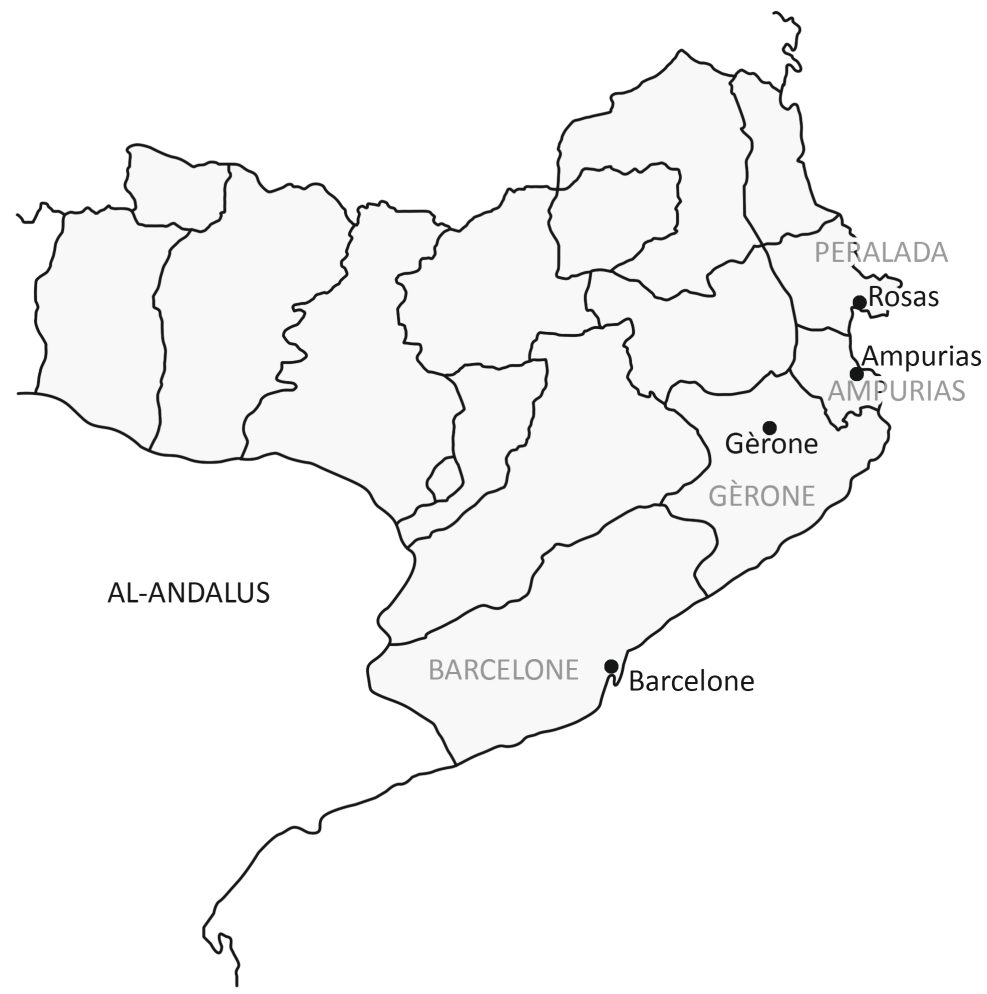

Figure 7 - Ateliers de frappe en Marche d'Espagne pendant le règne de Charlemagne.

Cet atelier frappa de la monnaie à l'époque wisigothe, sous le nom de Rodas ${ }^{58}$, avec ceux de Narbonne, Gérone et Barcelone. Tous quatre continuèrent à fonctionner sous la domination carolingienne, bien que l'identification de la Roda wisigothe avec la Roda carolingienne ait été remise en question par M. Crusafont. Selon lui, le manque d'importance de l'établissement à l'époque carolingienne et sa proximité avec Ampurias, atelier actif sous Charlemagne et Louis le Pieux, poussaient à envisager d'autres localisations possibles, comme Roda de Ter, près de Vic, ou Roda de Isábena, dans le comté de Ribagorza. Cette dernière option est d'ailleurs

58. À l'époque wisigothe, l'atelier de Roda a frappé monnaie à deux périodes : tout d'abord sous les règnes de Léovigilde, Récarède et Wittéric de la fin du vie siècle à l'an 610, et ensuite, de 687 à 710, sous les règnes d'Égica et Witiza. À l'époque d'Achila, le dernier roi wisigoth ayant gouverné dans cette zone, les ateliers de Gérone et de Narbonne ont continué à fonctionner, mais on ne connaît aucune émission de Barcelone ou de Rosas. 
celle qu'il considéra à un moment comme la plus probable ${ }^{59}$. Toutefois, le peu d'envergure de ce lieu à l'époque qui nous occupe semble contredire cette hypothèse. En effet, ce n'est qu'à partir de l'année 957 qu'il gagne en importance, une fois élevé au rang de diocèse par les comtes de Pallars et Ribagorza ${ }^{60}$, au détriment d'Urgell. Les découvertes monétaires dans la zone de l'Ampurdan, où se situe Rosas, la préexistence de l'atelier wisigoth et sa localisation dans un comté différent de celui d'Ampurias, celui de Peralada, appuient la thèse de l'identification de la Roda carolingienne avec le site de Rosas ${ }^{61}$. Actuellement la localisation à Rosas n'est plus guère discutée.

Le nombre de pièces carolingiennes connues de l'atelier de Roda était, jusqu'à la découverte d'El Tolmo de Minateda, limité à trois, deux sans provenance certaine : une monnaie de Louis le Pieux conservée au Cabinet des Médailles de la Bibliothèque nationale de France à Paris et la pièce de Charlemagne publiée par M. Crusafont à laquelle nous nous sommes intéressée précédemment ; et une obole de Louis le Pieux trouvée dans le Rhône à une quinzaine de kilomètres au sud de Lyon ${ }^{62}$. Il convient d'ajouter à celles-ci l'annonce faite par F. Mateu ${ }^{63}$ de la découverte à Rosas d'une pièce carolingienne de Louis le Pieux du même atelier «semblable à celle décrite par Botet I, p. 11 num. $8 »{ }^{64}$. C'est une découverte intéressante qui, si elle est validée, pourrait constituer un indice supplémentaire pour confirmer l'identification de cet atelier puisque cette pièce a été retrouvée sur son lieu présumé de frappe. À ces découvertes, trois certaines et une encore douteuse, s'ajoute à présent la monnaie retrouvée sur le site d'El Tolmo de Minateda.

Si l'on s'en tient aux preuves numismatiques, la production de l'atelier de Roda, ainsi que celle des autres ateliers de la Marche d'Espagne, a dû être très limitée ${ }^{65}$. Le faible nombre de pièces parvenues jusqu'à nous semble suggérer un volume réduit d'émissions, et, selon A.M. Balaguer ${ }^{66}$, les besoins de monnaie ont dû être couverts par des deniers carolingiens frappés dans des ateliers au nord

59. La discussion sur les possibles localisations de cet atelier dans CRUSAFONT 1981-1983, 1982, p. 29 et 1983, p. 131. Récemment BÉNÉZET et al. 2011.

60. Palol 1999, p.393.

61. Crusafont 1981-1983, p. 103. Selon A. Balaguer, le comté de Peralada devait revêtir une importance bien précise à l'époque carolingienne car il est mentionné à maintes reprises dans les sources écrites. Voir BALAGUER 1999, p. 27.

62. Sur cette obole voir BÉNÉZET et al. 2011.

63. Mateu 1946, p. 13, n. 24.

64. La pièce décrite par J. Botet est celle conservée au Cabinet des Médailles de la Bibliothèque nationale de France. Cette découverte n'a pas été retenue car elle était considérée comme peu fiable, tant par M. Crusafont (1983, n. 19) lors de sa publication d'un type inédit de ce même atelier, que par A.M. Balaguer (1984, addenda) dans son recueil de découvertes de monnaies carolingiennes en Catalogne. Elle l'a reconsidérée par la suite (BALAGUER 1999, p. 37).

65. Le recueil de monnaies d'ateliers hispaniques réalisé par M. Crusafont en 1982 ne contenait que 7 monnaies de Charlemagne et 19 de Louis le Pieux.

66. Balaguer 1987-1988a, 326 et 1999, p. 29 et 34. 
des Pyrénées, pour certains, très productifs ${ }^{67}$. Parmi les monnaies carolingiennes mises au jour dans la péninsule ibérique, il est assez fréquent de retrouver des pièces produites dans des ateliers du Languedoc et de la Provence, ce qui pousse M. Crusafont à supposer à l'existence d'une certaine forme de commerce ${ }^{68}$. Toutefois, M. Crusafont et A. Balaguer pensent, étant donné le manque de découvertes de monnaies frappées en la Marche d'Espagne, leur faible poids et leur style, que la présence de monnaies carolingiennes dans ce territoire pourrait attester non pas l'existence d'échanges, qui devaient être bien rares, mais plutôt le mouvement des armées franques. Suivant cette idée, ils ont proposé l'hypothèse qu'il s'agissait d'émissions à caractère belliqueux trouvant leur origine dans les différentes campagnes militaires des armées carolingiennes sur le territoire péninsulaire. La conquête de Barcelone en 801 ou les campagnes menées dans la zone de Tortose par Louis le Pieux expliqueraient, selon eux, pourquoi la plupart des pièces frappées dans des ateliers de la Marche d'Espagne ont été retrouvées sur le territoire français : les troupes les auraient en effet ramenées avec elles lors de leur retour dans leur lieu d'origine ${ }^{69}$. Cet argument n'est en revanche pas valable pour l'atelier de Roda, car on connaît pour le moment un seul exemplaire retrouvé au nord des Pyrénées : une obole de Louis le Pieux trouvée dans le Rhône à une quinzaine de kilomètres au sud de Lyon $^{70}$. En outre, comme le signale M. Crusafont ${ }^{71}$, l'existence d'émissions de cet atelier durant deux règnes, celui de Charlemagne et celui de Louis le Pieux, indique qu'il ne s'agit pas d'un atelier éphémère à caractère militaire, mais d'un atelier ayant perduré.

\section{La circulation de monnaie carolingienne dans la péninsule ibérique}

Les découvertes de monnaies carolingiennes dans la péninsule ibérique ont mis en évidence essentiellement trois zones de circulation : la frange septentrionale, l'Andalousie et la Catalogne actuelle ${ }^{72}$. Celles de la zone nord proviennent de fouilles archéologiques et présentent une chronologie tardive, située entre la fin du Xe siècle et le début du XIe siècle, époque à laquelle la présence franque dans cette zone se fait sentir essentiellement à travers l'ordre de Cluny et l'organisation des chemins de pèlerinage vers Saint-Jacques-de-Compostelle. Quatre pièces sont parvenues jusqu'à nous : deux deniers au type carolingien au nom de Guillaume Sanche de Bordeaux, mis au jour à l'ermitage de Santa Elena à Irun, un denier de

67. Un nombre significatif de pièces de l'atelier de Melle a été retrouvé en péninsule Ibérique. On en connaît $8 ; 5$ d'entre elles l'ont été en Catalogne, et les 3 autres à Irun, à Saint-Jacquesde-Compostelle et aux alentours de Séville, respectivement. Sur ces trouvailles et la bibliographie correspondante, voir BALAGUER 1999, p. 36-38, et CluA 2008, p. 81.

68. Crusafont 2004, p. 234.

69. Balaguer 1999, p. 34-35 ; Crusafont 1983, p. 129 et Crusafont, JoAnot 2009, p. 236.

70. BÉNEZET et al. 2011.

71. CRUSAFOnT 1981-1983, p.103.

72. Recueil des découvertes jusqu'en 1999, avec la bibliographie correspondante et leur localisation graphique dans BALAGUER 1999, p. 36-39. 
Melle portant le nom de Charles trouvé à Ibañeta, en Navarre, et un autre denier du même atelier découvert lors de fouilles à la cathédrale de Saint-Jacquesde-Compostelle.

Les monnaies de la zone andalouse sont des pièces isolées découvertes par hasard, et des pièces thésaurisées dans des dépôts de monnaie arabe. Frappées au IXe siècle, elles sont plus anciennes que celles de la zone septentrionale. Il y a trois pièces isolées, deux d'origine incertaine : un fragment de monnaie de Louis le Pieux au type Christiana Religio, qui se trouvait incrusté dans un dirham de l'émir andalou 'Abd al-Raḥman II de 230 H/840 apr. J.-C. en guise de métal pour compléter le poids de la pièce ${ }^{73}$; et une obole d'un type inédit, attribuée à Pépin II d'Aquitaine d'après l'existence d'un denier au même type déjà attribué à ce monarque ${ }^{74}$. La troisième pièce isolée est un fragment de denier portant le nom d'Eudes, émis à Angers et retrouvé dans la région de Séville ${ }^{75}$. La monnaie thésaurisée est beaucoup plus abondante et a été retrouvée dans deux trésors. Le premier, connu comme Sagrada Familia, a été retrouvé à Cordoue. Il contenait vingt fragments et trois pièces entières de monnaie carolingienne : cinq deniers de Louis le Pieux, un denier et une obole attribués à Charles le Chauve, de l'atelier de Toulouse, un autre denier au nom de Charles de Chalon-sur-Saône, ainsi que quelques pièces non attribuées de l'atelier d'Arles ${ }^{76}$. L'autre a été mis au jour à Puebla de Cazalla (Séville) et il contenait cinq pièces carolingiennes : un denier et trois fragments appartenant à Louis le Pieux dont l'atelier n'a pas été précisée, et un autre très petit fragment de Charles le Chauve frappé à Laon ${ }^{77}$.

C'est, en toute logique, dans les territoires situés au sud des Pyrénées sous domination franque, que les découvertes ont eu lieu en plus grand nombre. Alors qu'on n'en comptait encore aucune dans les années 80 du siècle dernier, on en compte aujourd'hui une douzaine, ayant eu lieu fortuitement, aussi bien de pièces isolées que d'ensembles ${ }^{78}$. À celles-ci s'ajoutent quelques exemplaires mis au jour plus récemment au cours de fouilles archéologiques menées dans la ville de Barcelone, parmi lesquels une obole de Louis le Pieux d'atelier non déterminé, et deux deniers et une obole de transition portant le nom de Charles retrouvés à place de Sant Miquel ${ }^{79}$, ainsi qu'un denier de Melle attribué à Charles le Chauve et une obole en mauvais état de conservation, probablement de Louis le Pieux ou de Pépin d'Aquitaine (817-838), provenant des fouilles des Archives municipales de Barcelone ${ }^{80}$.

73. Balaguer, CANTo 1987.

74. CRUSAFOnT 1987-1988.

75. BALAGUER 1987, 1988b.

76. Balaguer, Canto 1987, p. 46.

77. CANTO, IBRAHIM 1991.

78. BALAGUER 1984, 1987-1988a et 1999.

79. Marot 1999, p. 310. Ces deniers de transition, connus depuis longtemps, sont considérés émissions épiscopales par X. Sanahuja (2010, p. 94).

80. Clua 2008,p. 81. 
Si nous regroupons par règnes ces découvertes ayant eu lieu sur le territoire sous domination carolingienne au sud des Pyrénées, nous ne retrouvons que deux deniers émis lors du règne de Charlemagne : le premier près d'Ampurias et l'autre à Anserall dans l'Alto Urgell. De Louis le Pieux, le roi le mieux représenté pour le moment, on compte quatre pièces sûres, et possiblement une cinquième, retrouvées respectivement à Cerdanyola ${ }^{81}$, Rosas, Barcelone et dans un lieu indéterminé de la Catalogne. En ce qui concerne Charles le Chauve, six exemplaires mis au jour dans différents lieux de la Catalogne actuelle lui seraient dues. Bien que certaines pièces au type du monogramme lui aient été facilement attribuées du fait de leur style plus tardif, les autres pourraient appartenir également au règne de Charlemagne. Parmi les pièces plus tardives, il convient de mentionner deux oboles de Charles le Gros (884-887), la première, frappée à Toulouse, retrouvée au barrage de Sant Ponç (Solsonès) ${ }^{82}$, et l'autre, du même atelier, apparue dans un lieu indéterminé de la Catalogne ${ }^{83}$; trois pièces de l'atelier d'Arles attribuées à Louis l'Aveugle de Provence (887-928), retrouvées, respectivement, entre Tàrrega et Bellpuig ${ }^{84}$, à proximité de Lérida ${ }^{85}$ et dans un lieu indéterminé ${ }^{86}$; et, enfin, trois deniers d'Eudes (887-898), dont un de l'atelier de Toulouse apparu entre Tàrrega et Bellpuig ${ }^{87}$ et deux de Toulouse et Péronne, découverts dans un lieu imprécis ${ }^{88}$.

En dehors de ces trois zones, on connaît un denier de Melle au nom de Charles qui faisait partie d'un dépôt de monnaie normande et melgorienne mis au jour à Palma de Majorque ${ }^{89}$, ainsi qu'un denier carolingien et des fragments de deux autres, dont l'attribution n'a pas été précisée, mis au jour à Calatrava la Vieja $(\text { Ciudad Real })^{90}$. À ceux-ci s'ajoute à présent celui exhumé à El Tolmo de Minateda, établissement également éloigné des trois principales zones de découvertes précédemment citées, confirmant ainsi sa position au cœur d'un important réseau routier. Cette pièce était en circulation sous l'émirat.

81. J. Colomer (1984, p. 268) identifia cette pièce comme un denier. Toutefois, son poids de $0,77 \mathrm{~g}$ et son module de $15,5 \mathrm{~mm}$ indiquent qu'il s'agit d'une obole, comme le signale A. Balaguer (1984 et 1999) qui, par erreur, affirme dans la première de ses publications qu'elle est issue de l'atelier de Carcassonne alors que sur l'image de la pièce fournie par J. Colomer on peut clairement lire Narbonne, attribution exacte et mentionnée comme telle dans ses publications ultérieures.

82. Balaguer 1987-1988b, p. 320-321. Type Prou 822.

83. Crusafont 1987. Type Prou 822.

84. Ce lieu se trouvait en territoire musulman mais tout près des comtés chrétiens rattachés à l'Empire carolingien. Selon A. Balaguer $(1999,34)$, ces pièces mettent en évidence les contacts entre les deux zones.

85. CRUSAFont 2002, 2004.

86. Balaguer 1987-1988 a, p. 325. Type Prou 822 var.

87. BALAGUER 1998, p. 236.

88. BAlAguer 1987-1988a, p. 325. Type Prou 824 var. et 825 var. respectivement.

89. Selon A.M. Balaguer (1999, p. 38 no 26 et p. 109 n 5) ce denier de Melle présente «un type immobilisé datant du début du XIe siècle».

90. CANTO 2002, p. 115. 


\section{Monnaie carolingienne et monnaie islamique}

Le phénomène de l'apparition de deniers carolingiens aux côtés de pièces arabes est connu depuis longtemps, aussi bien sur le territoire andalou que dans le territoire franc. Les dépôts dans lesquels les deux monnaies apparaissent conjointement sont relativement nombreux et attestent des contacts entre les deux zones. J. Duplessy répertoriait en 1956 jusqu'à douze trésors mis au jour en Europe occidentale, datant du vIII au Xe siècle, qui contenaient des pièces islamiques mélangées à des deniers carolingiens, lombards et anglo-saxons ${ }^{91}$. Aujourd'hui, on en connaît quelques autres, comme ceux d'Ilanz et Steckborn en Suisse, celui de Muizen en Belgique, ou celui de Cuerdale en Angleterre (Lancashire) qui contenait quelques pièces d'al-Andalus ${ }^{92}$. Les monnaies islamiques et carolingiennes ne furent pas seulement thésaurisées conjointement, elles circulèrent également en même temps dans certaines régions. En témoigne la découverte à l'emplacement d'un ancien marché médiéval à Torcello, à Venise, de deux monnaies : un denier de Charlemagne de l'atelier de Milan et un dirham abbasside daté à la fin du IIe siècle de l'Hégire (800-820 apr. J.-C. $)^{93}$. Les deux monnaies sont apparues collées l'une à l'autre, avec encore des morceaux de tissu de la bourse qui les contenait, perdue dans ce marché. Pour le territoire franc, il convient tout particulièrement de mentionner la découverte, dans un tombeau carolingien de Contres (Loir-et-Cher), d'un dirham d'al-Andalus de 161 H./777-778 apr. J.-C., de l'émir “Abd al-Raḥman I ${ }^{\mathrm{er}}$, qui avait été coupé à la taille d'un denier carolingien ${ }^{94}$.

Dans la péninsule ibérique, sont documentés plusieurs trésors de monnaies arabes en argent, qui contenaient également des pièces carolingiennes. Dans tous ceux-ci, la monnaie franque apparaissait souvent fragmentée, représentant un pourcentage très réduit du total de l'argent thésaurisé ${ }^{95}$. Le premier mis au jour fut celui connu sous le nom de Sagrada Familia, provenant d'un quartier de la ville de Cordoue $^{96}$. Il contenait 170 pièces entières, une grande quantité de fragments et quelques morceaux d'autres objets, totalisant 1355 grammes d'argent. Parmi les monnaies se trouvaient vingt deniers carolingiens, trois entiers et dix-sept fragments, de Louis le Pieux et de Charles le Chauve ${ }^{97}$. S. Santos estime sa date d'enfouissement à 911 apr. J.-C., hypothèse remise en cause par A. Canto, qui le situe pour sa part à la décennie précédente sur la base des autres découvertes connues ${ }^{98}$.

91. DuPLESSY 1956, p. 101-163.

92. À propos de ces découvertes et d'autres mises au jour, voir Mc KorMICK 2005, p. 759-774.

93. Мс КоRмicк 2005, p. 306 et 774.

94. LoNGPÉrIER 1863 et MC KorMicK 2005, p. 329 et 762.

95. CANTO 2012, p. 72.

96. Santos 1956, Morrison, Grunthal 1967, p. 378 no 106, et Marcos, Vicent 1992, p. 202.

97. Selon A. Canto (2002, p. 115), il y aurait également des monnaies carolingiennes de Carloman (879-884).

98. CANTo 2002, p. 116. 
Puebla de Cazalla (Séville) est un autre trésor qui contenait des pièces carolingiennes. Il était composé de 266 dirhams entiers, de 636 dirhams fragmentés, d'un anneau en argent portant une inscription arabe et d'un fragment tubulaire en argent. Aux côtés de ces monnaies omeyyades, on a retrouvé deux dirhams abbassides, trois dirhams d'ateliers d'Afrique du Nord et cinq pièces carolingiennes de Louis le Pieux et de Charles le Chauve ${ }^{99}$. La date la plus tardive figure sur un dirham de 278 H./891-892 apr. J.-C., ce qui situe le moment de l'enfouissement vers la fin du IXe siècle ou le début du siècle suivant.

Du trésor de Calatrava la Vieja (Ciudad Real), nous savons seulement qu'il était composé de plus de cent dirhams de l'émirat, au milieu desquels se trouvaient un denier carolingien et des fragments de deux autres ${ }^{100}$. Enfin, nous avons connaissance de la découverte non pas d'un trésor mais d'une monnaie isolée : un fragment de denier de Louis le Pieux, inséré dans un dirham andalou de 230 H./844 apr. J.-C. avec une finalité métrologique. Le poids avait probablement été ajusté afin de convenir à certaines activités ou transactions ${ }^{101}$.

D'après S. Santos, la présence de monnaies carolingiennes au milieu de monnaies islamiques dans le trésor cordouan de Sagrada Familia serait due au fait qu'elles faisaient partie du butin conquis par les Arabes dans les territoires francs ${ }^{102}$. Selon les sources, Hišām I ${ }^{\mathrm{er}}$ avait obtenu de la razzia de 793 en Septimanie 45000 livres d'or pur, qui lui permirent de financer le premier agrandissement de la mosquée de Cordoue ${ }^{103}$. Cette interprétation, validée par d'autres auteurs ${ }^{104}$, expliquerait la présence de monnaies carolingiennes en Al-Andalus comme le fruit du butin obtenu lors des razzias sarrasines sur le territoire franc et non comme le fruit de relations commerciales. Cette explication ne vaut toutefois pas pour les trésors plus tardifs. La présence de monnaie carolingienne dans des trésors d'al-Andalus est égalment attestée durant le Califat de Cordoue au xe siècle. Deux de ceux-ci en témoignent : le premier, découvert en 1866 dans un lieu non précisé de la péninsule ibérique, contenait des deniers de Charles le Gros (884-898) et d'Eudes (887-898), des deniers et des oboles de Charles le Simple (898-923), ainsi que des émissions datant du xe siècle, attribuées à Raoul de Bourgogne (923-936), Guillaume de Brioude et Lothaire $(954-986)^{105}$. Le second, trouvé à

99. Selon A. Canto et T. Ibrahim (1991, p. 73), un denier type Morrison Grunthal 472, Grierson $3^{\mathrm{e}}$ classe ; trois fragments apparemment similaires au précédent; et un fragment de $0,20 \mathrm{~g}$ à peine, de l'atelier de Laon, similaire aux types MEC 860-870.

100. CANTO 2002, p. 115 et Martin et al. 2011.

101. CANTO, BaLAguer 1987.

102. SANTOS 1956, p. 85.

103. SÉNAC 2002, p. 65.

104. Par exemple Balaguer 1979, p. 300.

105. Découverte recueillie par GARIEL 1883-1884, vol. I, p. 136 et mentionnée par MARTíN et al.2011, p. 188-190, d'où nous avons tiré les données des exemplaires carolingiens identifiés. $X$. Sanahuja a étudié 49 monnaies apparues sur le marché numismatique. Il estime qu'elles peuvent appartenir à ce même trésor. Il n'y aucune monnaie de Lothaire parmi celles-ci. Les douze deniers de Guillaume (atelier de Brioude) datent l'ensemble d'entre 910 et 925 (Sanahuja 2010). 
Cordoue et connu comme «Haza Carmen ${ }^{106}$, est celui qui présente le plus grand ensemble de monnaie franque connu pour le moment dans la péninsule ibérique. Il s'agit d'un grand dépôt d'environ 7000 pièces, entières et fragmentées, datant de 320 H/932 apr. J.-C. à 386 H./996 apr. J.-C. Les exemplaires omeyyades d'al-Andalus étaient accompagnées de monnaies fatimides, d'autres abbassides très abîmées, et de 140 fragments de pièces chrétiennes également très détériorées et de dimensions réduites. Parmi ces fragments, des émissions des rois Eudes (887-898), Charles le Simple (898-923), Raoul de Bourgogne (923-936) et Louis IV (936-954) ${ }^{107}$ ont été identifiées. On peut ainsi voir que, bien que minoritaire, la monnaie carolingienne est une des composantes des enfouissements monétaires musulmans dans la péninsule ibérique pendant le Ixe et le xe siècle. La présence de ce numéraire ne peut pas s'expliquer seulement par les razzias sarrasines en territoires francs, mais il devait y avoir certains types de contact qui ont conduit à l'introduction de numéraire franc dans le territoire du Califat de Cordoue.

Les données disponibles sur les trouvailles de monnaies arabo-islamiques en Gaule méridionale permettent de préciser les relations existant entre celle-ci et al-Andalus. À partir du mode de diffusion de ces pièces aux marges de l'empire carolingien, deux zones se différencient clairement : la Septimanie, caractérisée par l'abondance des fulus de cuivre, et l'Aquitaine qui voit circuler des dirhams d'argent. Se confirme ainsi l'existence durant les vIIIe et IXe siècles d'un flux de ces monnaies arabo-islamiques vers le nord des Pyrénées et leur circulation réelle, voire leur utilisation en Gaule carolingienne ${ }^{108}$.

\section{Conclusion}

On ne connaissait la présence d'aucune monnaie attribuée à Charlemagne en péninsule ibérique jusqu'à la publication de deux pièces : la première, de l'atelier de Roda, trouvée près d'Ampuries, et l'autre, de celui de Toulouse, mise au jour à Anserall, dans l'Alto Urgell. Ces deux découvertes furent le fruit du hasard et nous ne disposons pas de données suffisantes sur les circonstances de ces trouvailles. L'absence de contexte archéologique connu a obligé à fonder leur attribution sur des critères stylistiques et historiques. À ces deux monnaies s'ajoute à présent la pièce d'El Tolmo de Minateda, que nous avons pu dater aussi du règne de Charlemagne. Il s'agit de la première monnaie d'un atelier hispanique retrouvée en dehors de la Marche d'Espagne et de l'un des rares exemplaires connus qui soit issu de celui de Roda. Pour son attribution, nous nous sommes fondée, non seulement sur les critères stylistiques et historiques classiques, mais également sur les données fournies par la position de la pièce dans la séquence stratigraphique du site.

106. Marcos, Vicent 1992, p. 212-214 ; CANTo 2002, p. 120, 2007, p. 31-37 et sous presse ; RuIz 2002.

107. Une étude détaillée des monnaies chrétiennes de ce trésor est disponible dans RuIz 2002.

108. Cette question a été avancée par J. Duplessy (1956) et développée récemment par M. Parvérie (2007, 2010 et 2012). Voir aussi BÉNEZET et al. 2003. 
Dans la mesure où la pièce provient d'un niveau archéologique en place, l'information fournie par le contexte est déterminante: une couche d'utilisation d'époque islamique, probablement établie dans une période proche de la phase de construction de la cité musulmane, à la fin du viIIe siècle ou plus probablement au début du Ixe siècle. On peut alors penser que cette monnaie carolingienne a été perdue à un certain moment de la première moitié du Ixe siècle. Cette donnée permet de proposer son attribution au règne de Charlemagne. L'absence sur le site de toute monnaie frappée au cours de la seconde moitié du IXe siècle, moment où la cité fut abandonnée, confirmerait bien cette hypothèse. La réduction du nombre d'ateliers de frappe carolingiens dans la Marche d'Espagne entamée avec Louis le Pieux apporte un autre argument puisque, pour le moment, les émissions de Charles le Chauve ne sont connues que pour celui de Barcelone.

La pièce de Charlemagne perdue dans une rue du quartier islamique d'El Tolmo de Minateda et recueillie en contexte archéologique, faisait partie de la monnaie ayant cours normal dans la cité musulmane, jusqu'à sa perte fortuite. Elle a dû être utilisée conjointement avec les dirhams et, comme eux, elle a été fragmentée, donnant lieu à une fraction régulière et reconnaissable de demi-pièce. Cette découverte atteste de la circulation simultanée de la monnaie carolingienne et de la monnaie arabe dans la péninsule ibérique, jusqu'alors mise en évidence uniquement par des trésors monétaires. Si cette monnaie carolingienne était en circulation, cela indique que, comme d'autres provenant de l'extérieur de la péninsule, elle y était acceptée comme moyen de paiement et n'était pas seulement thésaurisée pour sa valeur intrinsèque.

Le contexte stratigraphique de la pièce mise au jour à El Tolmo de Minateda suggère de plus une chronologie d'utilisation légèrement antérieure à celle proposée jusqu'à aujourd'hui en ce qui concerne l'arrivée de la monnaie carolingienne dans la péninsule ibérique. Elle constitue ainsi l'un des exemples plus anciens de circulation de celle-ci au sud des Pyrénées.

\section{Bibliographie}

ABAD 1996 : L. AbAD CASAL, La epigrafía del Tolmo de Minateda (Hellín, Albacete) y un nuevo municipio romano del Conventus Carthaginensis, Archivo Español de Arqueología, 69, 1996, p. 77-108.

Abad et al. 1998 : L. Abad Casal, S. Gutierrez Lloret, R. Sanz Gamo, El Tolmo de Minateda. Una historia de tres mil quinientos años, Toledo, 1998.

Abad et al. 2012 : L. Abad Casal, S. Gutierrez Lloret, Bl. Gamo Parras, P. Canovas Guillen, El tolmo de Minateda, un proyecto de investigación y puesta en valor del patrimonio, Debates de Arqueología Medieval, 2, 2012.

Amoros et al. 2012 : V. Amoros Ruiz, V. Canavate Castejon, S. Gutierrez Lloret, J. Sarabia Bautista, Cerámica altomedieval en el Tolmo de Minateda (Hellín, Albacete, España), IX Congresso Internazionale AIECM2, Venecia, 2009, p. 245-256. 
Balaguer 1979 : A.M. Balaguer Prunes, Primeres conclusions de l'estudi de la moneda catalana comtal, Symposium Numismático de Barcelona, vol. II, Barcelona, p. 297-327.

Balaguer 1981-1983 : A.M. Balaguer Prunes, Els dos tallers monetaris de nom Roda o Rodas a la Hispània visigoda, Lambard, Barcelona, 1981-1983 (II), p. 105-107.

Balaguer 1983 : A.M. Balaguer Prunes, El problema de la localización de la Roda visigoda, Acta Numismática, 13, 1983, p. 109-118.

Balaguer 1984 : A. M. Balaguer Prunes, Troballes de moneda carolingia a Catalunya, Gaceta Numismática, 74/75, 1984, p. 143-146.

Balaguer 1987-1988a : A.M. Balaguer Prunes, Les troballes de moneda carolingia a l'àmbit Peninsular, Acta Numismática, 17/18, 1987-1988, p. 324-330.

Balaguer 1987-1988b : A.M. Balaguer Prunes, Troballes monetàries VII, Acta Numismática, 17-18, 1987-1988, p. 317-323.

Balaguer 1998 : A.M. Balaguer Prunes, Troballes monetàries XIV, Acta Numismática, 28, 1998, p. 235-238.

B Alaguer 1999 : A.M. B Alaguer Prunes, Història de la moneda dels Comtats Catalans, Barcelona, 1999.

Balaguer et al. 1979 : A.M. Balaguer Prunes, M. Crusafont, I. Puig, Els comtats catalans: les seves encunyacions i àrees d'influencia. I. Corpus. Symposium Numismático de Barcelona, vol. I, Barcelona, 1979, p. 377-508.

Balaguer, Canto 1987 : A.M. Balaguer Prunes, A. Canto Garcia, Al-Andalus y los carolingios, un singular testimonio monetario, Gaceta Numismática, 85 , 1987, p. 41-49.

BÉNÉZet et al. 2011 : J. BÉNÉzet, Ph. Schiesser, J.-F. Letho-Duclos, Une obole de Rosas au nom de Louis le Pieux. BSFN, 2011, 7, p. 190-192.

Botet 1908-1911 : J. Bотет I Siso, Les monedes catalanes, Barcelona, 19081911.

Canto 2002 : A. Canto Garcia, Moneda foránea en al-Andalus, X Congreso Nacional de Numismática (Albacete, 1998), Madrid, 2002, p. 107-128.

CAnto 2007 : A. CAnto Garcia, Haza del Carmen (Córdoba, 1981), Maskukat. Tesoros de monedas andalusíes en el Museo Arqueológico de Córdoba, Córdoba, 2007, p. 31-37.

Canto 2012 : A. Canto Garcia, Al-Andalus: dinero, monedas y medios de intercambio, dans Ph. Sénac (éd.), Histoire et Archéologie de l'occident musulman (VIIe-XVe siècle : al-Andalus, Maghreb, Sicile), CNRS - Université de Toulouse II-Le Mirail, Toulouse, 2012, p. 67-79.

Canto, Ibrahim 1991 : A. Canto Garcia, T. Ibrahim, Hallazgo emiral en Puebla de Cazalla (Sevilla), Numisma, 229, 1991, p. 69-85.

CAnto, sous presse : A. CAnto GARCiA, El hallazgo de moneda califal de Haza del Carmen (Córdoba).

Clua 2002 : M. Clua i Mercadal, Circulació monetària des de l'època medieval a les comarques de Barcelona, Barcelona, 2002. 
Clua 2008 : M. Clua i Mercadal, La cultura material en Barcelona y su Hinterland en época de Almanzor: Las monedas, dans J. Luis del Pino (coord.), La península Ibérica al filo del año 1000, Congreso Internacional Almanzor y suépoca (Córdoba, 14 al 18 de octubre de 2002), Córdoba, 2008, p. 78-82.

COSTER 1852 : L. de Coster, Restitution de quelques monnaies à Charlemagne, $R B N, 1852$, p. 369-403.

Coupland $1988:$ S. Coupland, Dorestad in the ninth century: the numismatic evidence, Jaarboek voor Munt-en Penningkunde, 75, 1988, p. 5-26.

Coupland 2002 : S. CouPLAND, Trading places: Quentovic and Dorestad reassessed, Early Medieval Europe, 11, 2002, p. 209-232.

Coupland 2005 : S. Coupland, Charlemagne's coinage: ideology and economy, dans J. Story (ed.), Charlemagne: Empire and Society, Manchester University Press, 2005, p. 211-229.

Coupland 2007 : S. Coupland, Carolingian coinage and the Vikings: studies on power and trade in the $9^{\text {th }}$ century, Variorum Collected Studies, Aldershot, 2007.

Coupland 2010-2011 : S. Coupland, Carolingian Single Find and the Economy of the Early Ninth Century, NC, 170, 2010, p. 287-sqq.

Coupland 2010-2012 : S. Coupland, Boom and bust at $9^{\text {th }}$-century Dorestad, dans A. Willemsen, H. Kik (eds.), Dorestad in an international framework: new research on centres of trade and coinage in Carolingian times (Proceedings of the first Dorestad Congress held at the National Museum of Antiquities) (the Netherlands June 24-27), Leiden, 2009.

Crusafont 1980 : M. Crusafont i Sabater, Nou tipus carolingi de Barcelona de Carles el Calb, II Simposi Numismàtic de Barcelona, Barcelona, 1980, p. 47-55.

Crusafont 1981-1983 : M. Crusafont i Sabater, Carlemany i el taller monetari de Roda, Lambard, Barcelona, 1981-1983 (II), p. 101-103.

Crusafont 1982 : M. Crusafont i Sabater, Numismática de la Corona CatalanoAragonesa Medieval (785-1516), Madrid, 1982.

Crusafont 1983 : M. Crusafont i Sabater, Tipo inédito de Carlomagno de la ceca de Roda, Acta Numismática, 13, 1983, p. 125-135.

Crusafont 1987 : M. Crusafont i SABAter, Consecuencias de dos hallazgos de monedas catalano-carolingias de transición, Mélanges offerts au Dr.J.-B. Colbert de Beaulieu, París, 1987, p. 227-234.

Crusafont 1987-1988: M. Crusafont i SABater, Troballa a Andalusia, Troballes monetàries VII, Acta Numismática, 17-18, 1987-1988, p. 321-322.

Crusafont 1996 : M. Crusafont i SABATER, Història de la moneda catalana, Barcelona, 1996.

Crusafont 2002 : M. Crusafont i Sabater, Dinero inédito carolingio de Arlés atribuible a Carlos el ciego, $R N, 158,2002$, p. 271-278.

Crusafont 2004 : M. Crusafont i Sabater, Troballa de Lleida Ciutat, Acta Numismática, 34, 2004, p. 233-234. 
Crusafont 2009 : M. Crusafont i SABAter, Catàleg general de la moneda catalana: països catalans i corona catalano-aragonesa (s. v a.C.- s. XX d.C.), Barcelone, 2009.

Crusafont, Janot 2009 : M. Crusafont i Sabater, A. Janot i B Arrull, Troballa d'Anserall, Acta Numismática, 39, 2009, p. 233-236, 239.

DePEYrot 2008 : G. DePEYRot, Le numéraire carolingien. Corpus des monnaies, (Moneta 77), Wetteren, 2008 (3 éd. augmentée).

Domenech 2002 : C. Domenech Belda, Trientes de Witiza, La Lección Tiempo. Arte y Cultura en Castilla-La Mancha, Toledo, 2002, p. 58-59.

Domenech 2009 : C. Domenech Belda, Numismática y arqueología medieval: la moneda de excavación y sus aportaciones, dans A. Arévalo (ed.), XIII Congreso Nacional de Numismática (Cádiz 22-24 octubre 2007), 2009, p. 707-736.

Domenech 2010 : C. Domenech Belda, El proceso de islamización en el Sharq al-Andalus a través de los registros monetales, dans P. Sénac (éd.), Villa 3: Histoire et Archéologie des sociétes de la Vallée de L'Ebre (VIIe-XIe siècles), CNRS - Université de Toulouse II-Le Mirail, 2010, p. 275-293.

Domenech, Gutierrez 2005 : C. Domenech Belda, S. Gutierrez Lloret, Las monedas del Tolmo de Minateda (Hellín, Albacete), XIII Congreso Internacional de Numismática (Madrid, 2003), Madrid, 2005, p. 1567-1576.

Domenech, Gutierrez 2006 : C. Domenech Belda, S. Gutierrez Lloret, Viejas y nuevas monedas en la ciudad emiral de Madīnat Iyyuh (El Tolmo de Minateda, Hellín, Albacete), Al-Qantara, XXVII- 2, p. 337-374.

DuPLESSY 1956 : J. DuPLESSY, La circulation des monnaies arabes en Europe Occidentale du vIII au XIII' siècle, $R N, 1956$, p. 101-163.

Engel, Serrure 1890 : A. Engel, R. Serrure, Traité de Numismatique du Moyen Âge, t. 1, Paris, 1890.

GARIEL 1883-1884 : E. GARIEL, Les monnaies royales de France sous la race carolingienne, Strasbourg, 1883-1884.

Grierson 1965 : Ph. GrIERSON, Money and coinage under Charlemagne, Karl der Grosse, vol. I, Düsseldorf, 1965, p. 501-536.

Grierson, Blackburn 1986 : Ph. Grierson, M. Blackburn, Early Middle Ages $\left(5^{\text {th }}-10^{\text {th }}\right.$ centuries $),($ Medieval European Coinage 1), Cambridge, 1986.

Gutierrez 2000 : S. Gutierrez Lloret, La identificación de Madīnat Iyyuh y su relación con la sede episcopal Elotana. Nuevas perspectivas sobre viejos problemas, Scripta in Honorem E. A. Llobregat, Alicante, 2000, p. 481-501.

Gutierrez 2011 : S. Gutierrez Lloret, El Tolmo de Minateda en torno al 711, 711. Arqueología e Historia entre dos mundos, Zona arqueológica, 15, vol. I, 2011, p. 355-374.

Gutierrez et al. 2003 : S. Gutierrez Lloret, Bl. Gamo Parras, V. Amoros Ruiz, Los contextos cerámicos altomedievales del Tolmo de Minateda y la cerámica altomedieval en el sureste de la Península ibérica, dans L. Caballero,P. Mateos, M. Retuerce (ed.), Cerámicas tardorromanas y altomedievales en la Península Ibérica. Ruptura y continuidad, (Anejos de Archivo Español de Arqueología XXVIII), p. 119-168. 
Gutierrez et al. 2005 : S. Gutierrez Lloret, L. Abad Casal, Bl. Gamo Parras, Eio, Iyyuh y El Tolmo de Minateda (Hellín, Albacete) de sede episcopal a Madina islámica, VI Reunió d'Arqueologia Cristiana Hispànica, Barcelona, 2005, p. 345-370.

Gutierrez, Canovas 2009 : S. Gutierrez Lloret, P. Canovas Guillen, Construyendo el siglo VII : arquitecturas y sistemas constructivos en el Tolmo de Minateda, dans P. Mateos, M.A. Utrero (ed.), El siglo VII frente al siglo VII. Arquitectura, (Anejos de Archivo Español de Arqueología XLVIII), p. 91-131.

Gutierrez, Domenech, sous presse : S. Gutierrez Lloret, C. Domenech BeldA, Coinage, Context and Social Space. The High Medieval city of El Tolmo de Minateda (Hellín, Albacete, Spain), I Workshop Internazionale di Numismatica: 'Numismatica e Archeologia. Monete, stratigrafie e contesti. Dati a confronto' (Roma, 28-30 settembre 2011).

LAFAURIE 1952 : J. LAFAURIE, Le trésor monétaire du Puy (Haute-Loire). Contribution à l'étude de la monnaie de la fin du xe siècle, $R N, 1952$, p. 59-169.

LAFAURIE 1976 : J. LAfAURIE, Moneta palatina, Francia, 4, 1952, p. 59-87.

LeChUGA 2000 : M. LeChuga Galindo, Una aproximación a la circulación monetaria de época tardía en Cartagena: los hallazgos del teatro romano, V Reunió d'Arqueologia cristiana Hispànica (Cartagena, 1998), Barcelona, 2000, p. 333-349.

Lechuga, Mendez 1991 : M. Lechuga Galindo, R. Mendez Ortiz, Numismática bizantina de Cartagena, Historia de Cartagena, VI, Murcia, 1991, p. 72-78.

LONGPERIER 1863 : A. de LONGPERIER, Monnaie andalouse trouvée à Contres, $R N$, 1863 , p. 214-216.

Marcos et Vicent 1992 : A. Marcos Pous, A. M. Vicent Zaragoza, Los tesorillos de moneda hispano-árabe del Museo Arqueológico de Córdoba, III Jarique de Numismática Hispano-Árabe (Madrid, 1990), Madrid, 1992, p. 183-218.

Martin et al. 2011 : F. Martin Escudero, J. Minguez Martinez, A. Canto Garcia, La circulación monetaria en el reinado de Alfonso III a través de las fuentes documentales, dans A. García Leal, R. Gutiérrez González, C.E. Prieto Entrialgo (ed.), MC aniversario de la muerte de Alfonso III y de la tripartición del territorio del Reino de Asturias, t. II, p. 157-205.

Marot 1999 : T. MARot i SALSAS, La moneda en época carolingia: reflejo de una realidad histórica, dans Cataluña en la época carolingia. Catálogo de exposición, Barcelona, 1999, p. 49-51.

Mateu 1946 : F. MAteAu i Llopis, De la Hispania tarraconense visigoda a la Marca Hispánica carolina, Analecta Sacra Tarraconensis, XIX, 1946, p. 1-122.

Mc CoRMick 2005 : M. Mc CoRmick, Orígenes de la economía europea. Viajeros y comerciantes en la Alta Edad Media, Barcelona, 2005.

Morrison, Grunthal 1967 : K.F. Morrison, H. Grunthal, Carolingian Coinage, New York, 1967.

Palol 1999 : P. de Palol (dir.), Del Romà al romànic, Barcelona, 1999.

PARVÉRIE 2007 : M. PARVÉRIE, La circulation des monnaies arabes en Aquitaine et Septimaine, VIIIe-IXe siècles, Aquitania, 23, 2007, p. 233-246. 
Parvérie 2010 : M. PARvérie, La circulation des dirhams d'al-Andalus entre Gascogne et Aquitaine au IXe siècle, BSFN, juin 2010, p. 144-149.

PARVÉRIE 2012 : M. PARVÉRIE, Questions sur l'importation de dirhams d'al-Andalus dans l'empire carolingien, BCEN, 49/1, 2012, p. 14-23.

Prou 1892 : M. Prou, Les monnaies carolingiennes, Paris, 1892, 1969 (rééd.). Ruzz 2002 : Gl. Ruz GarCia, Monedas cristianas en un hallazgo califal, X Congreso Nacional de Numismática (Albacete, 1998), Madrid, 2002, p. 491-500.

Sanahuja 2006 : X. Sanahuja Anguera, La moneda de Barcelona al segle X, segons les troballes Espanya-1 i Espanya-2 (925), Acta Numismática, 36, 2006, p. 79-113.

Santos 1956 : S. de los Santos Gener, Monedas carolingias en un tesorillo de dirhemes del Emirato cordobés, Numario Hispánico, 5, 1956, p. 80-87.

SARAH 2010 : G. SARAH, Charlemagne, Charles the Bald and the Karolus monogram coinage. A multi-disciplinary study, $N C, 170,2010$, p. 227-286.

Sarah et al. 2011 : G. Sarah, J. Le Gall, Y. Menez, Les sites d'occupation du haut Moyen-Âge de Saint-Symphorien et de Bressilien à Paule (Côtes d'Armor, France). Synthèse des campagnes 2008-2010 et étude pluridisciplinaire du matériel monétaire, The Journal of Archaeological Numismatics, 1, 2011, p. 217-236.

SÉNAC 2002 : Ph. SÉNAC, Les carolingiens et al-Andalus (VIIIe-IXe siècles), Paris, 2002.

SILLIÈRES 1982 : P. SilLIÈRES, Une grande route romaine menant à Carthagène : la voie Saltigi-Carthago Nova, Madridder Mitteilungen, 23, 1982, p. 247-257.

Sillières 1990 : P. Sillières, Les voies de communication de l'Hispanie méridionale, Paris, 1990. 

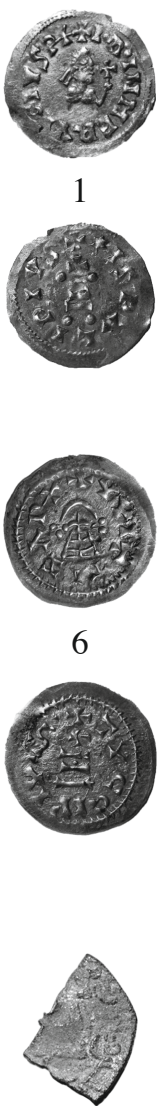

11
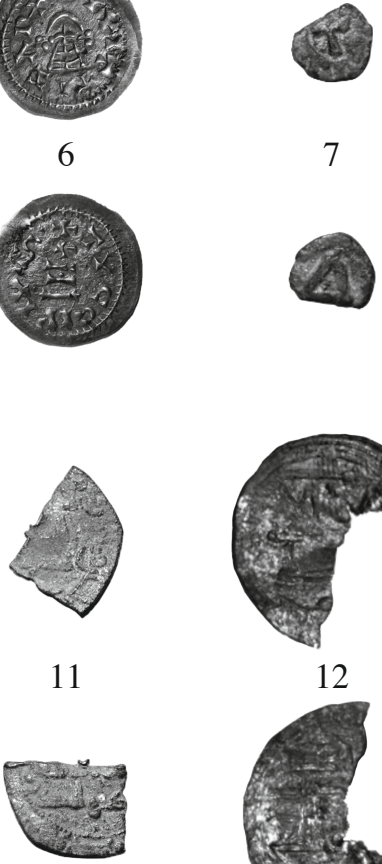

7
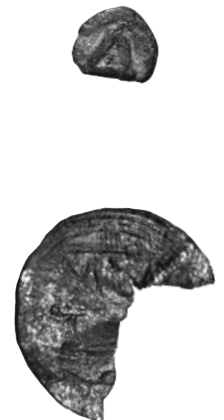

12
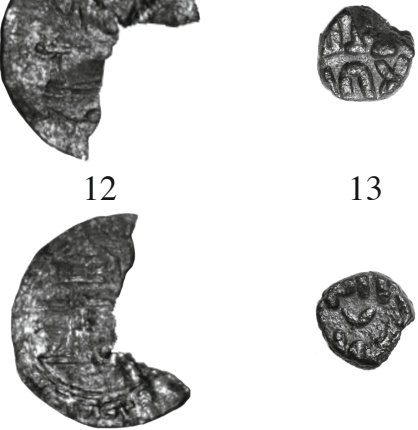

13

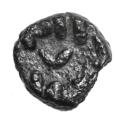

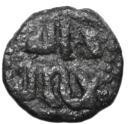

14

15
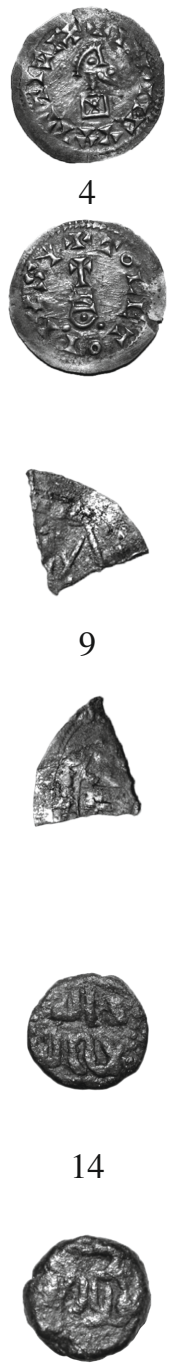

4

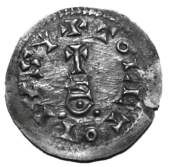

5
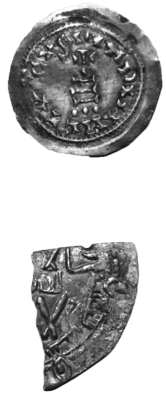

10
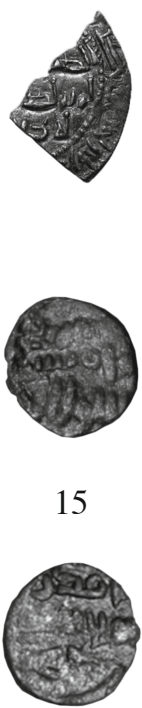

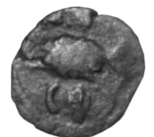

16

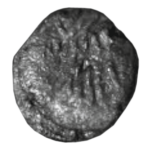

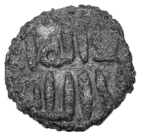

18
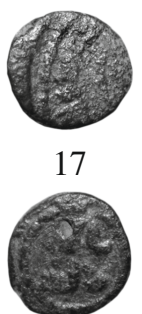

17

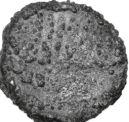

